\title{
ENTROPIES OF AUTOMORPHISMS OF A TOPOLOGICAL MARKOV SHIFT
}

\author{
D. A. LIND
}

\begin{abstract}
Let $\sigma$ be a mixing topological Markov shift, $\lambda$ a weak Perron number, $q(t)$ a polynomial with nonnegative integer coefficients, and $r$ a nonnegative rational. We construct a homeomorphism commuting with $\sigma$ whose topological entropy is $\log [q(\lambda) q(1 / \lambda)]^{r}$. These values are shown to include the logarithms of all weak Perron numbers, and are dense in the nonnegative reals.
\end{abstract}

1. Introduction. Let $A$ be a square nonnegative integral matrix. We assume throughout that $A$ is aperiodic in that some power of $A$ is strictly positive. A familiar procedure [Wi] associates to $A$ a homeomorphism $\sigma_{A}$ of a totally disconnected compact space $X_{A}$. Aperiodicity of $A$ means that $\sigma_{A}$ is topologically mixing. Such homeomorphisms are called topological Markov shifts or subshifts of finite type. For a discussion of their central role in dynamics, see [DGS].

Denote by aut $\left(\sigma_{A}\right)$ the group of homeomorphisms of $X_{A}$ commuting with $\sigma_{A}$. The case $A=[n]$ was studied by Hedlund and coworkers $[\mathbf{H}]$, whose principal motivation appears to have been cryptographic. The algebraic complexity of aut $\left(\sigma_{[n]}\right)$ is indicated by Hedlund's theorem that it contains an isomorphic copy of every finite subgroup. The proof easily extends to general $\sigma_{A}$. Although aut $\left(\sigma_{A}\right)$ has been recently studied by several authors [Wa, BK, BLR], its algebraic structure remains mostly a mystery. No one knows, for example, whether $\operatorname{aut}\left(\sigma_{[2]}\right)$ is generated by $\sigma_{[2]}$ and the involutions in the group.

By the Curtis-Lyndon-Hedlund theorem $[\mathbf{H}]$, each $\varphi \in \operatorname{aut}\left(\sigma_{A}\right)$ is induced by a block map. Thus the topological entropy $h(\varphi)$ is finite. Such an automorphism $\varphi$ can be regarded as a bijective cellular automaton map [Wo], and $h(\varphi)$ measures the information flow of the map. What are the possible values of $h(\varphi)$ for $\varphi \in \operatorname{aut}\left(\sigma_{A}\right)$ ? Denote this set by $H_{A}$. Despite the finite nature of shift commuting maps, there are essentially no nontrivial exact computations of their entropy in the literature. One exception is Coven's calculation of the entropy of a map commuting with the one-sided 2-shift $[\mathbf{C}]$. In particular, it was unknown whether or not $H_{A}$ is dense in $[0, \infty)$.

Our purpose here is to construct automorphisms of $\sigma_{A}$ with interesting entropies. We will obtain values, like $\log (9 / 2)$, that are sometimes not logarithms of algebraic integers, and which form a dense subset of $[0, \infty)$.

To state the precise results, call $\lambda$ a Perron number (and write $\lambda \in \mathbf{P}$ ) if $\lambda \geq 1$ is an algebraic integer that strictly exceeds the absolute value of its other conjugates.

Received by the editors December 12, 1985.

1980 Mathematics Subject Classification (1985 Revision). Primary 54H20; Secondary 58F11, $68 \mathrm{Q} 80$.

Key words and phrases. Topological entropy, Perron number, automorphism group of a topological Markov shift.

The author was supported in part by NSF grant DMS-8320356. 
Call $\lambda \geq 1$ weak Perron $(\lambda \in W \mathbf{P})$ if some nonnegative integral power of it is Perron. Let $\mathbf{Q}^{+}=\{r \in \mathbf{Q}: r \geq 0\}$ and $\mathbf{Z}^{+}=\mathbf{Z} \cap \mathbf{Q}^{+}$. In $[\mathbf{L}]$ it is shown that $\mathbf{P}$ coincides with the set of spectral radii of aperiodic $\mathbf{Z}^{+}$-matrices, and $W \mathbf{P}$ is the set of spectral radii of arbitrary $\mathbf{Z}^{+}$-matrices. Let

$$
\Lambda=\left\{\left[q(\lambda) q\left(\lambda^{-1}\right)\right]^{r}: \lambda \in W \mathbf{P}, q(t) \in \mathbf{Z}^{+}[t], r \in \mathbf{Q}^{+}\right\} .
$$

In Theorem 1 we show that if $\alpha \in \Lambda$, there is a $\varphi \in \operatorname{aut}\left(\sigma_{A}\right)$ with $h(\varphi)=\log \alpha$. In Theorem 2 we prove that $W \mathbf{P} \subset \Lambda$. Thus our results are summarized by

$$
\log W \mathbf{P} \subset \log \Lambda \subset H_{A}
$$

for every aperiodic $A$.

The construction uses markers to define two involutions in aut $\left(\sigma_{A}\right)$. Their composition moves buffers that reserve differing lengths of space for storing information. This movement causes background data from an auxiliary topological Markov shift to be shifted by various amounts. The whole marker-buffer-data system is topologically conjugate to a certain skew product whose entropy can be calculated using results of Marcus and Newhouse [MN], leading to the numbers above. One consequence is the existence of elements in $\operatorname{aut}\left(\sigma_{A}\right)$ with exactly two measures of maximal entropy. This construction is also used in [BLR] to embed the countable direct sum of infinite cyclic groups into the automorphism group of every mixing topological Markov shift.

Most of the ideas used in our construction are contained in $\S 2$. There we find, for fixed $q(t) \in \mathbf{Z}^{+}[t]$, an automorphism of a special full shift whose entropy is $\log \left[q(2) q\left(\frac{1}{2}\right)\right]$. In $\S 3$ we show how to adapt and elaborate these ideas to prove Theorem 1. Some elementary estimates in $\S 4$ prove Theorem 2.

The author thanks the Mathematical Sciences Research Institute and the IBM Thomas J. Watson Research Center for their generous hospitality during visits in which part of this work was done.

2. Constructing automorphisms. Suppose $q(t) \in \mathbf{Z}^{+}[t]$. We construct an automorphism $\varphi$ of the full $q(1)+3$ shift with $h(\varphi)=\log \left[q(2) q\left(\frac{1}{2}\right)\right]$.

If $q(t)=q_{d} t^{d}+\cdots+q_{1} t+q_{0}$ with $q_{i} \in \mathbf{Z}^{+}$, let

$$
L=\{0,1, m\} \cup\left\{b_{i j}: 0 \leq i \leq d, 1 \leq j \leq q_{i}\right\} .
$$

If $q_{i}=0$, there are no symbols $b_{i j}$. Let $\sigma_{L}$ denote the full $L$-shift on $X_{L}$. Using juxtaposition to denote concatenation of symbols, create buffers of length $i+1$ from each $b_{i j}$ by putting $B_{i j}=b_{i j}^{i+1}$. We use $i+1$ rather than $i$ to allow several buffers when $i=0$.

Next we define two involutions $\varphi_{1}$ and $\varphi_{2}$ in $\operatorname{aut}\left(\sigma_{L}\right)$. Consider blocks of the form $m B_{i j} D B_{r s} m$ with data $D \in\{0,1\}^{2 d-i-r+3}$. For such blocks $|D| \geq 3$, where $|D|$ denotes the length of the string $D$. We declare that $\varphi_{1}$ replaces $m B_{i j} D B_{r s} m$ with $m B_{r s} D B_{i j} m$ and vice versa, has no other effect. Clearly $\varphi_{1}^{2}=I$, the identity, so $\varphi_{1} \in \operatorname{aut}\left(\sigma_{L}\right)$. The definition of $\varphi_{2}$ is more complicated. Let a frame be a block of the form

$$
m B_{i_{0} j_{0}} D_{0} B_{r_{0} s_{0}} m^{*} B_{i_{1} j_{1}} D_{1} B_{r_{1} s_{1}} m
$$

where $D_{k} \in\{0,1\}^{2 d-i_{k}-r_{k}+3}(k=0,1)$, and ${ }^{*}$ denotes the central symbol. We declare $\varphi_{2}$ to be a block map acting only on frames, and taking the above frame to $D_{10} B_{i_{1} j_{1}} m^{*} B_{r_{0} s_{0}}$ if $r_{0} \geq i_{1}$, where $D_{1}=D_{10} D_{11}$ with $D_{10} \in\{0,1\}^{r_{0}-i_{1}}$, and to 
$B_{i_{1} j_{1}} m^{*} B_{r_{0} s_{0}} D_{01}$ if $r_{0} \leq i_{1}$, where $D_{0}=D_{00} D_{01}$ with $D_{01} \in\{0,1\}^{i_{1}-r_{0}}$. Since $\varphi_{2}$ does not disturb markers and affects only symbols within $d+1$ of $m^{*}$, while markers in a frame are spaced $2 d+3$ apart, it follows that $\varphi_{2}$ is well defined. Although $\varphi_{2}$ can affect the entries in a frame, it does not affect the property of being a frame. Hence $\varphi_{2}^{2}=I$, and $\varphi_{2} \in \operatorname{aut}\left(\sigma_{L}\right)$.

Let $\varphi=\varphi_{2} \varphi_{1}$. To compute $h(\varphi)$, we first construct a $\varphi$-invariant compact set $Y$ such that $h(\varphi)=h\left(\left.\varphi\right|_{Y}\right)$. We then observe that $\left.\varphi\right|_{Y}$ is topologically conjugate to a certain skew product, whose entropy we compute using $[\mathbf{M N}]$.

Let

$$
Y=\left\{x \in X_{L}: x=\left(\cdots m B_{i_{-1} j_{-1}} D_{-1} B_{r_{-1} s_{-1}} m^{*} B_{i_{0} j_{0}} D_{0} B_{r_{0} s_{0}} m \cdots\right)\right\},
$$

where ${ }^{*}$ denotes the 0 th coordinate, and

$$
D_{n} \in\{0,1\}^{2 d-i_{n}-r_{n}+3}, \quad n \in \mathbf{Z} .
$$

Then $\varphi$ acts on $Y$ as follows. The buffer $B_{i_{n} j_{n}}$ is shifted right to replace $B_{i_{n+1} j_{n+1}}$, and $B_{r_{n} s_{n}}$ is shifted left to replace $B_{r_{n-1} s_{n-1}}$. The background data in $D_{0}$ is shifted by $i_{0}-r_{0}$ to the left by $\varphi_{1}$, and not shifted by $\varphi_{2}$. Thus near the 0 th coordinate, the background data in the infinite string $\cdots D_{-1} D_{0} D_{1} \cdots$ maintains its order and is shifted by $i_{-n+1}-r_{n-1}$ under $\varphi^{n}$.

If the defining pattern for $Y$ is broken, buffers shifting towards the break are reflected to return in the opposite direction. Background data near such a break can move by at most a bounded amount for all iterates of $\varphi$.

To initiate the computation of $h(\varphi)$, let $A$ partition $X_{L}$ by the 0 th coordinate. For $p \geq 0$ let

$$
A^{p}=\bigvee_{|j| \leq p} \sigma_{L}^{j}(A)
$$

where empty intersections are discarded. Then

$$
h\left(\varphi, A^{p}\right)=\lim _{N \rightarrow \infty} \frac{1}{N} \log \left|\bigvee_{j=0}^{N} \varphi^{-j}\left(A^{p}\right)\right|,
$$

and since $A$ generates under $\sigma_{L}$,

$$
h(\varphi)=\lim _{p \rightarrow \infty} h\left(\varphi, A^{p}\right) .
$$

We assume $p>3 d+6$, and estimate the cardinality of $\bigvee_{j=0}^{N} \varphi^{-j}\left(A^{p}\right)$ as follows. For $x \in X_{L}$ define $x[a, b]$ to be the block $x_{a} x_{a+1} \cdots x_{b}$. Say a block $B$ occurs in a subshift $Z \subset X_{L}$ (and write $B \in Z$ ) if there is a $z \in Z$ and $a, b$ so that $z[a, b]=B$. First suppose $F$ is a nonempty atom of $\bigvee_{j=0}^{N} \varphi^{-j}\left(A^{p}\right)$, and that for $x \in F$ we have $x[-p, p] \notin Y_{0}=\bigcup_{k=0}^{2 d+5} \sigma_{L}^{k} Y$. By the description of the action of $\varphi$ given above, we see that $x[-p, p]$ contains a break acting as a reflecting barrier to buffers and data. It follows that $\left(\varphi^{j} x\right)[-p, p]$ can only contain new information from a possible new buffer entering from the right, another entering from the left, and a bounded amount of data. Since there are $q(1)$ different buffers, we obtain

$$
\left|\bigvee_{j=0}^{N} \varphi^{-j}\left(\mathcal{A}^{p}\right) \cap Y_{0}^{c}\right|=O\left(q(1)^{2 N}\right) .
$$


Thus $h\left(\left.\varphi\right|_{Y_{0}^{c}}\right) \leq \log q(1)^{2}$. Now $h\left(\left.\varphi\right|_{Y_{0}}\right)=\max \left\{h\left(\left.\varphi\right|_{\sigma_{L}^{k} Y}\right): 0 \leq k \leq 2 d+5\right\}=$ $h\left(\left.\varphi\right|_{Y}\right)$, and we will show that $h\left(\left.\varphi\right|_{Y}\right)=\log \left[q(2) q\left(\frac{1}{2}\right)\right]$. Hence the evaluation of $h(\varphi)$ follows from the next result.

Lemma. Let $q(t) \in \mathbf{Z}^{+}[t]$. Then

$$
\frac{d^{2}}{d t^{2}}\left[q(t) q\left(\frac{1}{t}\right)\right] \in \mathbf{Z}^{+}\left[t, t^{-1}\right] .
$$

Hence $q(t) q(1 / t)$ is increasing and convex on $[1, \infty)$.

Proof. Put $f(t)=q(t) q(1 / t)=\sum_{j=-d}^{d} c_{j} t^{j}$, where $c_{j} \in \mathbf{Z}^{+}$. Then

$$
f^{\prime \prime}(t)=\sum_{j=-d}^{d} j(j-1) c_{j} t^{j-2} \in \mathbf{Z}^{+}\left[t, t^{-1}\right] .
$$

Since $f^{\prime}(1)=0$, the second statement follows.

To compute $h\left(\left.\varphi\right|_{Y}\right)$, consider $B=\left\{B_{i j}: 0 \leq i \leq d, 1 \leq j \leq q_{i}\right\}$ as a set of abstract symbols. Let $\sigma_{B}$ denote the shift on the space $X_{B}$ of all sequences of the $B_{i j}$. For $u=\left(B_{i_{k} j_{k}}: k \in \mathbf{Z}\right) \in X_{B}$ and $v=\left(B_{r_{k} s_{k}}: k \in \mathbf{Z}\right)$, define $g: X_{B} \times X_{B} \rightarrow \mathbf{Z}$ by $g(u, v)=i_{0}-r_{0}$. Define the skew product $\tau=\left(\sigma_{B} \times \sigma_{B}^{-1}\right) \times \sigma_{2}^{g}$ on $Z=$ $X_{B} \times X_{B} \times X_{2}$ by

$$
\tau(u, v, w)=\left(\sigma_{B} u, \sigma_{B}^{-1} v, \sigma_{2}^{g(u, v)} w\right) .
$$

Our next goal is to prove $\left.\varphi\right|_{Y}$ is conjugate to $\tau$.

Define $\theta: Y \rightarrow Z$ by

$$
\begin{aligned}
& \theta\left(\cdots m^{*} B_{i_{0} j_{0}} D_{0} B_{r_{0} s_{0}} m \cdots\right) \\
& \quad=\left(\left(\cdots B_{i_{0} j_{0}}^{*} B_{i_{1} j_{1}} \cdots\right),\left(\cdots B_{r_{0} s_{0}}^{*} B_{r_{1} s_{1}} \cdots\right),\left(\cdots D_{0}^{*} D_{1} \cdots\right)\right),
\end{aligned}
$$

where $D_{0}^{*}$ indicates the 0 th coordinate at symbol $d+1-i_{0}$ of $D_{0}$. Note that on the left side $B_{i_{k} j_{k}}$ denotes a block of symbols from $L$, while on the right it is considered as a single symbol from the alphabet $B$. Clearly $\theta$ is a continuous bijection from $Y$ to $Z$, and from our description of $\left.\varphi\right|_{Y}$ above it follows that $\theta \varphi=\tau \theta$, so $\theta$ conjugates $\left.\varphi\right|_{Y}$ with $\tau$.

To complete an analysis of $\varphi$, we use $[\mathbf{M N}]$ to compute $h(\tau)=h\left(\left.\varphi\right|_{Y}\right)$. By Theorem B of $[\mathbf{M N}]$,

$$
h(\tau)=\max \left\{P\left(\sigma_{B} \times \sigma_{B}^{-1},(\log 2) g\right), P\left(\sigma_{B} \times \sigma_{B}^{-1},-(\log 2) g\right)\right\},
$$

where $P$ denotes topological pressure. These pressures are explicitly computable, coincide, and by $[\mathbf{M N}$, p. 111] equal

$$
\begin{array}{r}
\log \left(\sum_{\left(B_{i j}, B_{r s}\right) \in B \times B} 2^{g\left(B_{i j}, B_{r s}\right)}\right) \\
=\log \left(\sum_{\left(B_{i j}, B_{r s}\right)} 2^{i-r}\right)=\log \left[\left(\sum_{B_{i j}} 2^{i}\right)\left(\sum_{B_{r s}} 2^{-r}\right)\right] \\
=\log \left[\left(\sum_{r=0}^{d} q_{i} 2^{i}\right)\left(\sum_{r=0}^{d} q_{r} 2^{-r}\right)\right]=\log \left[q(2) q\left(\frac{1}{2}\right)\right] .
\end{array}
$$

This proves $h(\varphi)=\log \left[q(2) q\left(\frac{1}{2}\right)\right]$. 
We conclude this section by indicating a modification to obtain an automorphism $\varphi$ with $h(\varphi)=r \log \left[q(2) q\left(\frac{1}{2}\right)\right]$ for $r \in \mathbf{Q}^{+}$. By using powers, it suffices to find, for given $n \geq 1$, an automorphism with entropy $(1 / n) \log \left[q(2) q\left(\frac{1}{2}\right)\right]$. Fixing $n$, replace the symbol $m$ with $n$ distinct symbols $m_{0}, m_{1}, \ldots, m_{n-1}$, expanding $L$ to

$$
\left\{m_{0}, m_{1}, \ldots, m_{n-1}, 0,1\right\} \cup\left\{b_{i j}: 0 \leq i \leq d, 1 \leq j \leq q_{i}\right\} .
$$

Define $\varphi_{1}$ and $\varphi_{2}$ in aut $\left(\sigma_{L}\right)$ as above with $m$ replaced by $m_{0}$. Define $\varphi_{3}$ to be the 1-block map $\varphi_{3}\left(m_{j}\right)=m_{j+1(\bmod n)}$. Put $\varphi=\varphi_{3} \varphi_{2} \varphi_{1}$. Let $Y$ be the compact set above, except with $m$ replaced by $m_{0}$. Then $\left.\varphi^{n}\right|_{Y}=\left.\varphi_{2} \varphi_{1}\right|_{Y}$, and estimates as before show $h\left(\left.\varphi^{n}\right|_{Y}\right)=h\left(\varphi^{n}\right)$. Hence

$$
n h(\varphi)=h\left(\varphi_{2} \varphi_{1}\right)=\log [q(2) q(1 / 2)],
$$

concluding the verification that $\varphi$ has the stated entropy.

3. Extension to general topological Markov shifts. The constructions in $\S 2$ took place on a special full shift. We show here how to imitate these ideas to construct automorphisms of a general mixing subshift of finite type, and how to employ background data from an arbitrary subshift of finite type instead of the 2 -shift. This yields the automorphisms described in $\S 1$.

THEOREM 1. Let $\sigma_{A}$ be a mixing topological Markov shift, $\lambda \in W \mathbf{P}, q(t) \in$ $\mathbf{Z}^{+}[t]$, and $r \in \mathbf{Q}^{+}$. There is an automorphism $\varphi$ of $\sigma_{A}$ with

$$
h(\varphi)=r \log [q(\lambda) q(1 / \lambda)] .
$$

PROOF. We will construct $\varphi \in \operatorname{aut}\left(\sigma_{A}\right)$ with $h(\varphi)=\log [q(\lambda) q(1 / \lambda)]$. By cycling markers and taking powers as in $\S 2$, it is possible to obtain this entropy multiplied by an arbitrary $r \in \mathbf{Q}^{+}$. The argument is the same, and will be omitted.

Since $\lambda \in W \mathbf{P}$, by $[\mathbf{L}]$ there is an irreducible $0-1$ matrix $E$ with spectral radius $\lambda$. Let $e=\operatorname{dim} E$, and set $N=q(1)+e+1$. We first find $N$ blocks of equal length in $X_{A}$ so that no (not necessarily distinct) pair can overlap at all, with the trivial exception that each block entirely overlaps itself.

Since $\sigma_{A}$ is mixing and nontrivial, there must be a loop $i_{0} i_{1} \cdots i_{k} i_{0} \in X_{A}$ of distinct symbols with $k \geq 1$. Furthermore, one of these symbols, which we can assume is $i_{0}$, is followed by a symbol $j_{1} \neq i_{1}$. First suppose $j_{1} \neq i_{0}$. Choose a path of minimal length from $j_{1}$ to the loop, say $j_{1} j_{2} \cdots j_{r} j_{s}$. The case $r=0$ is possible and corresponds to $j_{1}=i_{s}$ for some $s \neq 0,1$. Define $\alpha=i_{0} \cdots i_{k}$, $\beta=i_{0} j_{1} j_{2} \cdots j_{r} i_{s} \cdots i_{k}$. Then the $N$ blocks $\alpha^{2} \beta^{r+1} \alpha \beta^{N+1-r}$ for $1 \leq r \leq N$ have no nontrivial overlaps. If $j_{1}=i_{0}$, put $\alpha=i_{1} \cdots i_{r} i_{0}$, and use $\alpha^{2} i_{0}^{r} \alpha i_{0}^{\bar{N}}-r+1$ for $1 \leq r \leq N$.

Label these blocks by $\mu, \beta_{i j}\left(0 \leq i \leq d, 1 \leq j \leq q_{i}\right)$, and $\delta_{i}(0 \leq i \leq e-1)$, and put $\Delta=\left\{\delta_{i}: 0 \leq i \leq e-1\right\}$. Then every point in $X_{A}$ decomposes uniquely into nonoverlapping occurrences of these blocks, separated by other strings. Now define $\varphi=\varphi_{2} \varphi_{1}$ as follows. Put $B_{i j}=\beta_{i j}^{i+1}$. The automorphism $\varphi_{1}$ replaces $\mu B_{i j} D B_{r s} \mu$ with $\mu B_{r s} D B_{i j} \mu$ and vice versa, where $D=\delta_{i_{1}} \cdots \delta_{i_{k}}, k=2 d-i-$ $r+3$, and $i_{1} \cdots i_{k} \in X_{E}$. To construct $\varphi_{2}$, define a frame to be a block of the form $\mu B_{i_{0} j_{0}} D_{0} B_{r_{0} s_{0}} \mu^{*} B_{i_{1} j_{1}} D_{1} B_{r_{1} s_{1}} \mu$, where $D_{k} \in \Delta^{2 d-i_{k}-r_{k}+3}(k=0,1)$ and the subscripts of the $\delta$ 's appearing in $D_{0} D_{1}$ form a block in $X_{E}$. As in $\S 2, \varphi_{2}$ 
is defined to be a block map acting only on frames, and taking the above frame to $D_{10} B_{i_{1} j_{1}} \mu^{*} B_{r_{0} s_{0}}$ if $r_{0} \geq i_{1}$, where $D_{1}=D_{10} D_{11}$ with $D_{10} \in \Delta^{r_{0}-i_{1}}$, and to $B_{i_{1} j_{1}} \mu^{*} B_{r_{0} s_{0}} D_{01}$ if $r_{0} \leq i_{1}$, where $D_{0}=D_{00} D_{01}$ with $D_{01} \in \Delta^{i_{1}-r_{0}}$. Noting that such a block map preserves the frame property, including the condition that $D_{0} D_{1} \in X_{E}$, the reasoning of $\S 2$ applies to prove that $\Delta_{2}$ is a well-defined involution in $\operatorname{aut}\left(\sigma_{A}\right)$.

As in $\S 2$, set

$$
Y=\left\{x \in X_{A}: x=\left(\cdots \mu^{*} B_{i_{0} j_{0}} D_{0} B_{\tau_{0} s_{0}} \mu \cdots\right)\right\},
$$

where $\left|B_{i_{k} j_{k}} D_{k} B_{r_{k} s_{k}}\right|=(2 d+5)|\mu|$ for $n \in \mathbf{Z}$ and $\cdots D_{-1} D_{0} D_{1} \cdots \in X_{E}$. Arguing as before, $Y$ is $\varphi$-invariant, $h(\varphi)=h\left(\left.\varphi\right|_{Y}\right)$, and $\left.\varphi\right|_{Y}$ is conjugate to the skew product $\tau=\left(\sigma_{B} \times \sigma_{B}\right) \times \sigma_{E}^{g}$. The calculation of $h(\tau)$ in $[\mathbf{M N}]$ proves that

$$
h(\varphi)=h\left(\left.\varphi\right|_{Y}\right)=h(\tau)=P\left(\sigma_{B} \times \sigma_{B}^{-1},(\log \lambda) g\right)=\log [q(\lambda) q(1 / \lambda)],
$$

completing the proof.

4. Automorphisms with Perron entropies. The set $\Lambda$ defined in $\S 1$ is a collection of algebraic numbers dense in $[1, \infty)$. Which algebraic integers are in $\Lambda$ ? The following is a partial answer.

THEOREM 2. $W \mathbf{P} \subset \Lambda$.

Proof. The rough ideas are that for large $|t|$ the function $(1+t)\left(1+t^{-1}\right)$ is essentially $t+2$, and that if $\lambda \in \mathbf{P}$ has the absolute value of its other conjugates $<\lambda-4$, then $\lambda-2 \in \mathbf{P}$.

Specifically, let $\pi \in W \mathbf{P}$ and choose $k$ so that $\lambda=\pi^{k} \in \mathbf{P}$. Since $\Lambda$ is closed under taking roots, we need only find $n$ so that $\lambda^{n} \in \Lambda$. Let $\lambda$ have conjugates $\lambda_{1}=\lambda, \lambda_{2}, \ldots, \lambda_{d}$, where $\left|\lambda_{j}\right|<\lambda(j \geq 2)$. Choose $n$ large enough so $\lambda^{n}>\left|\lambda_{j}^{n}\right|+6$ $(j \geq 2)$. The roots of

$$
(1+t)\left(1+t^{-1}\right)=z
$$

are

$$
f_{ \pm}(z)=\frac{1}{2}\left(z-2 \pm \sqrt{z^{2}-4 z}\right)
$$

where $\sqrt{z}$ denotes the standard branch. We will show that $\alpha=f_{+}\left(\lambda^{n}\right)$ is Perron, which will prove that $\pi=\left[(1+\alpha)\left(1+\alpha^{-1}\right)\right]^{1 / n k} \in \Lambda$.

Since $\lambda$ is integral, (*) with $z=\lambda^{n}$ is monic in $t$ with integral coefficients, so $\alpha$ is integral. Note that

$$
\left|f_{ \pm}(z)\right| \leq \frac{1}{2}\left\{|z|+2+\sqrt{|z|^{2}+4|z|}\right\} \leq|z|+2 .
$$

Furthermore, since $4 /\left(\lambda^{n}-2\right)^{2}<1$,

$$
\begin{aligned}
\alpha=f_{+}\left(\lambda^{n}\right) & =\frac{1}{2}\left(\lambda^{n}-2\right)\left\{1+\sqrt{1-\frac{4}{\left(\lambda^{2}-2\right)^{2}}}\right\} \\
& \geq \frac{1}{2}\left(\lambda^{n}-2\right)\left\{2-\frac{4}{\left(\lambda^{n}-2\right)^{2}}\right\}>\lambda^{n}-3 .
\end{aligned}
$$

Now the other conjugates of $\alpha$ are either $f_{-}\left(\lambda^{n}\right)$ or have the form $f_{ \pm}\left(\lambda_{j}^{n}\right)(j \geq 2)$. Clearly $\left|f_{-}\left(\lambda^{n}\right)\right|<\alpha$, and since $\left|\lambda_{j}^{n}\right|+6<\lambda^{n}$ we have

$$
\left|f_{ \pm}\left(\lambda_{j}^{n}\right)\right| \leq\left|\lambda_{j}^{n}\right|+2<\lambda^{n}-4<f_{+}\left(\lambda^{n}\right)=\alpha,
$$

proving $\alpha \in \mathbf{P}$ and completing the proof. 


\section{REFERENCES}

[BK] M. Boyle and W. Krieger, Periodic points and automorphisms of the shift, Trans. Amer. Math. Soc. (to appear).

[BLR] M. Boyle, D. Lind and D. Rudolph, The automorphism group of a subshift of finite type, preprint, Universities of Maryland and Washington, 1986.

[C] E. Coven, Topological entropy of block maps, Proc. Amer. Math. Soc. 78 (1980), 590-594.

[DGS] M. Denker, C. Grillenberger and K. Sigmund, Ergodic theory on compact spaces, Lecture Notes in Math., vol. 527, Springer-Verlag, New York, 1976.

[H] G. Hedlund, Endomorphisms and automorphisms of the shift dynamical system, Math. Systems Theory 3 (1969), 320-375.

[L] D. Lind, The entropies of topological Markov shifts and a related class of algebraic integer, Ergodic Theory Dynamical Systems 4 (1984), 283-300.

[MN] B. Marcus and S. Newhouse, Measures of maximal entropy for a class of skew products, Lecture Notes in Math., vol. 729, Springer-Verlag, New York, 1978, pp. 105-124.

[R] J. Ryan, The shift and commutativity, Math. Systems Theory 6 (1978), 82-85.

[Wa] J. Wagoner, Markov partitions and $K_{2}$, preprint, University of California, Berkeley, 1985.

[Wi] R. Williams, Classification of subshifts of finite type, Ann. of Math. (2) 98 (1973), 120153; errata Ann. of Math. (2) 99 (1974), 380-381.

[Wo] S. Wolfram, Universality and complexity in cellular automata, Phys. D 10 (1984), 1-35.

Department of Mathematics, University of WAShington, Seattle, WashingTON 98195 